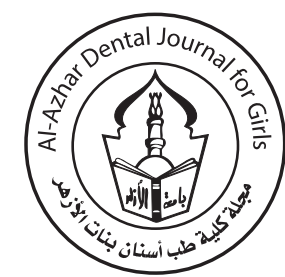

\title{
Experimental Study of Bone Defect Repair Using Centrifuged and Non-Centrifuged Bone Marrow Aspirate
}

\author{
Hanaa H El-Marzouky ${ }^{(1)}$, Fatma A Khalifa ${ }^{(2)}$, Ghada A Khalifa ${ }^{(3)}$, Ashraf A Shamaa ${ }^{(4)}$, \\ Mona H Farid ${ }^{(5)}$ and Shadia A. Elsayed ${ }^{(6)}$
}

Codex : 08/1901

azhardentj@azhar.edu.eg

http://adjg.journals.ekb.eg

DOI: $10.21608 /$ adjg.2019.5248.1003

\begin{abstract}
Purpose this study was conducted between 2015 and 2017 at the faculty of Veterinary Medicine Cairo University and aimed to histologically compare between the effect of processed and unprocessed bone marrow aspirate on the quantity and quality of the newly formed bone. Material and methods 72 Adult male white New Zealand rabbits were used in this study and were divided equally into 2 groups A and B. Bone defects were created at the medial upper extremity of right and left tibia of all rabbits down to bone using trephine bur measuring $4 \mathrm{~mm}$ diameter to $5 \mathrm{~mm}$ depth. The bone defects were treated with whole bone marrow loaded on gel foam in group A right tibias and using mononuclear cell layer obtained through centrifuging bone marrow loaded on gel foam in group B right tibias. Bone defects in the left tibias in all rabbits treated with gel foam only. Scarification was done 3,6,12 weeks postoperative. Histological evaluation was done using $\mathrm{H}$ and E and Masson's trichrome stain. Results the results showed that group A right tibial defects had better healing results than group B right tibial defects all over the follow-up period, but the differences were not statically significant. Conclusion the study findings indicate that the centrifugal concentration techniques provide better therapeutic outcomes over the whole BMA, but it is statistically insignificant.
\end{abstract}

\section{INTRODUCTION}

The limitations of autogenous allogenic and synthetic bone substitutes in augmenting critical size defects ${ }^{(1)}$ necessitate the development of other treatment modality. The field of biotechnology and tissue

\section{KEYWORDS}

bone, centrifuged, marrow, non-centrifuged, stem
Cairo, Egypt. 
engineering has offered new options for the repair of bony defects. . It depends on the use of mesenchymal stem cells (MSCs), which are multipotent adult stem cells of a mesodermal origin which have excellent renewal ability and osteogenic differentiation capabilities ${ }^{(2,3)}$. MSCs can be isolated from all connective tissues; however the bone marrow is the most common source for harvesting stem cells and progenitor cells due to its accessibility for surgeons and the extensive studies that have been done upon it. The iliac crest is documented to be the most common site for obtaining bone marrow via aspiration technique ${ }^{(4)}$.

The bone marrow aspirate can be used either processed (concentrated) or unprocessed. This processing (concentration) is done by the centrifugation of the aspirate, then harvesting the mononuclear cell (MNC) portion. The aim of this processing is to increase the number of mesenchymal stem cells and progenitor cells which subsequently increase the rate of bone healing ${ }^{(5-6)}$. However, there is still wide controversy regards the processing the bone marrow aspirate. Therefore this study was carried out to histologically compare between the use of centrifuged and non-centrifuged bone marrow aspirate on repairing bone defect.

\section{MATERIAL AND METHODS}

\section{Animal preparation:}

White male adult New Zealand rabbits weighting from 2.5 to 3 kilograms with an age range from 10 to 15 months were obtained from a local supplier. They were kept in the animal house under the veterinary supervision at animal housing of the Surgery Department, Faculty of Veterinary Medicine, Cairo University.

\section{Premedication and Anesthesia:}

All the operation were performed under general anesthesia using intramuscular injection of xylazine hydrochlorid $(5 \mathrm{mg} / \mathrm{kg})$ followed by ketamine hydrochlorid $(35 \mathrm{mg} / \mathrm{kg})$ these drugs served as sedative, anaesthetic and muscle relaxant.

\section{Surgical steps}

\section{Shaving and disinfection of surgical sites}

The skin over proximal part of femur and proximal medial part of both tibias were shaved and scrubbed with povidone iodine to reduce sepsis

\section{Bone marrow aspiration:}

Local anesthesia with vasoconstrictor (Mepecaine-LTM) was infiltrated into the periosteum and the local tissues for anesthesia and hemostasis. By using a sterile Rosenthal pediatric needle (18 gauge) applied at the trochanteric fossa $4.5 \mathrm{ml}$ bone marrow was aspirated into a plastic syringe containing $1 \mathrm{ml}$ sodium citrate anticoagulant.

\section{Bone marrow preparation}

The bone marrow aspirate was diluted 1:1 with phosphate buffered saline PBS. Then, $9 \mathrm{ml}$ of diluted marrow was layered onto $6 \mathrm{ml}$ lymphocyte separation medium expelled carefully along the wall of conical falcon tube containing Lymphocyte separation medium density $1.077 \mathrm{~g} / \mathrm{ml}$, so that the two layers do not mix together. The centrifugation was done at $400 \mathrm{x}$ g (gravitational force) for $15 \mathrm{~min}$ (3500 RPM). After centrifugation, the result will be RBCS layer at the bottom of the tube, lymphocyte separation medium layer with plasma/PBS layer on the top of it. The MNCs were harvested from the interface at the top of the lymphocyte separation medium layer.

\section{Bone defects creation and augmentation:}

The skin incision $2-3 \mathrm{~cm}$ in length was performed at the medial upper extremity of both tibias down to bone after injection of local anaesthesia with vasoconstrictor into the periosteum and the local tissues. Using a trephine bur measuring $4 \mathrm{~mm}$ the defects were created to $5 \mathrm{~mm}$ depth (Figure 1). All the defects in right tibias augmented with MNCs layer which was loaded on gel foam in group A. While those in group B were augmented with noncentrifuged (whole) bone marrow aspirate that was loaded on gel foams. On the other hand, the defects in left tibias in both groups were augmented with 


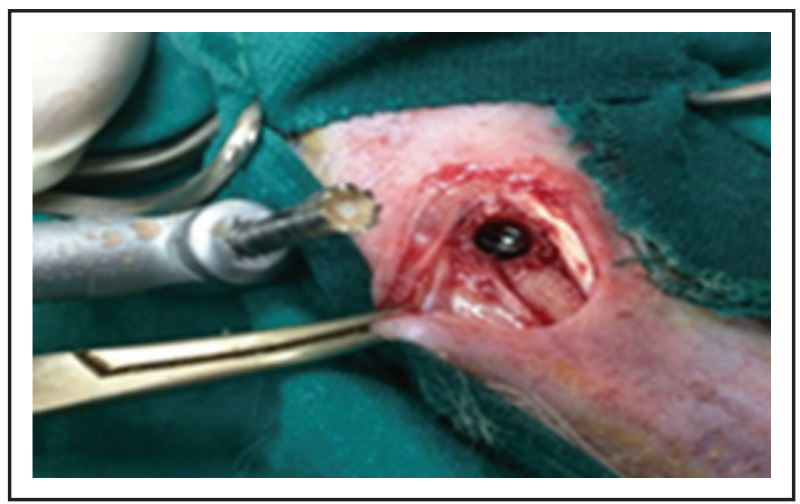

Fig. (1) The bone defect was created using trephine bur with dimensions of $4 \mathrm{~mm}$ width and $5 \mathrm{~mm}$ depth.

only gel foams. The wound was closed in layers by absorbable suture material.

\section{Post-operative care and follow up:}

Antibiotic (Cefteriaxon Sodium 1gm) was given in a dose of $0.1 \mathrm{ml} / \mathrm{kg}$ body weight per day via I.M. injection for 3 days to avoid postoperative infection.

\section{Sacrifice of animals:}

The sacrifices were performed at the 3rd, 6th, and 12 th weeks postoperatively. The specimens were harvested including the grafted defect area and about $4 \mathrm{~mm}$ of the adjacent normal bone and were placed in $10 \%$ Formalin fixative solution for maximum 24 hours.

\section{Histological examination:}

The specimens were submitted to histological processing, and they were stained by using $H \& E$ and Masson's trichrome stains. To measure the area percent of mature bone trabeculae digital images of Masson's trichrome stained sections were obtained. The area percent of mature bone trabeculae (stained red) was measured by using image $\mathbf{J}$ software as it identifies bone trabeculae areas according to their staining and then measures the area occupied by bone with respect to the total defect area examined.

\section{Statistical analysis:}

Values were presented as mean and standard deviation values. Data were explored for normal- ity using Kolmogorov-Smirnov test of normality. The One way analysis of variance ANOVA test was used to compare between groups at different times. This was followed by Tukey's post hoc test for pairwise comparison when the difference was found to be significant. The significance level was set at $\mathrm{p} \leq$ 0.05 . Statistical analysis was performed with SPSS 25.0 (Statistical Package for Scientific Studies, SPSS, Inc., Chicago, IL, USA) for Windows.

\section{RESULTS}

Twenty two rabbits died through the study period, and they were replaced by others. All the defects healed without any sign of infection or inflammation, and at the end of the follow up period all the defects were completely filled with bone.

\section{At 3 weeks healing period}

\section{With H\&E stain}

The right defects in group A revealed that the centers of defects were filled with granulation tissue (Figure2 A). At the periphery, newly formed bony spicules interconnected together. While in group B, the right tibial defects had their centers filled with cartilaginous tissue (Figure2 B). While, the left defects showed that the center of the defects were filled with granulation tissues and traces of cartilage. While their peripheries showed newly formed very thin and immature bony spicules.

\section{With Masson's trichrome stain}

In group A the bone trabeculae in the right tibia stained red with few amount of collagen that make negligible blue rim around osteocytes. While in the left defects both granulation tissue and cartilaginous area exhibit bluish stain. The newly formed bony spicules stained red beside traces of collagen that exhibit blue stain. In group B, the right tibial defect center filled with cartilage area which stained blue, and the periphery have newly formed bony spicules enclosing marrow spaces stained red with traces of collagen that stained blue 


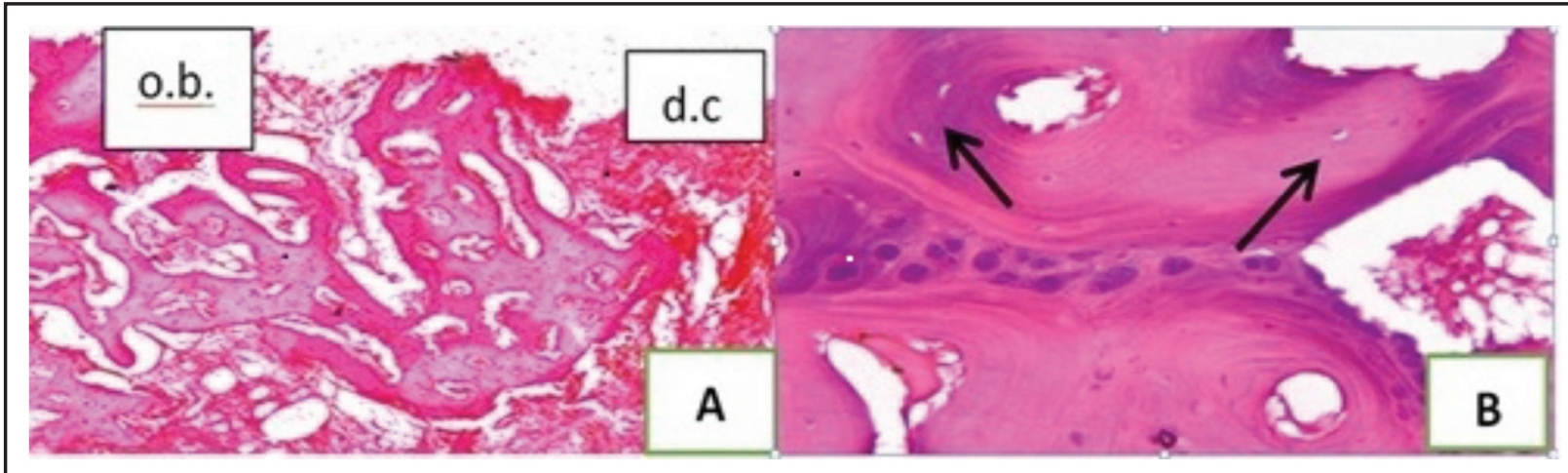

Fig. (2) A) a photomicrograph showing group A right defect H\&E (x40) 3 weeks. The defect center (d.c.) filled with granulation and fibrous tissue. At the periphery of defect just beside the old bone (o.b.) newly formed bony spicules inter connected to each other enclosing variable bone marrow spaces. B) a photomicrograph showing group B right tibial defect H\&E stain (x200) 3weeks. The cartilaginous area enclosed variable sized chondrocytes. Multiple newly formed endochondral bony spicules containing osteocytes in their lacunae (arrows) and enclosing different sizes marrow spaces while their peripheries showed newly formed bony spicules.

\section{At 6\& 12 weeks healing period}

\section{With H\&E stain}

The group A right defects showed formation of bone trabeculae with normal appearance and arrangement of osteocyte lacunae. However in group $\mathrm{B}$, the right defects had osteocyte lacunae with a relatively normal arrangement and some lacunae were empty (devoid of osteocytes nuclei. The left defects showed formation of osteocyte lacunae that had abnormal arrangement, and the most of the lacunae were devoid of osteocyte nuclei, and the present nuclei (if present) had abnormal eccentric appearance.

\section{With Masson's trichrome stain}

The group A right defects bone trabeculae stained red with few amount of collagen that only form negligible blue rim around lacunae. In group $\mathrm{B}$, the right defects bone trabeculae stained red with areas of collagen that acquire blue stain and form thicker blue rims around the lacunae. While the left defects bone trabeculae stained red with large areas of collagen which stained blue and form thicker blue rims around the lacunae. Using independent $t$ test in comparing between both groups $\mathrm{A}$ and group $\mathrm{B}$ right defects at 3,6, and 12 weeks healing period, revealed that the mean $\%$ area of mature bone difference was statistically insignificant (Table 1)

Table (1) Showing comparison of area percent of both groups A and group B right defects ( $t$ test).

\begin{tabular}{|c|c|c|c|c|}
\hline \multicolumn{2}{|r|}{ Time interval } & Group A & Group B & p- value \\
\hline \multirow[b]{2}{*}{3 weeks } & Mean $\%$ area of mature bone \pm standard deviation & $50.81 \pm 8.96$ & $40.22 \pm 7.19$ & \multirow{2}{*}{$.073^{\text {ns }}$} \\
\hline & Standard error mean & 4.00544 & 3.21609 & \\
\hline \multirow[b]{2}{*}{6 weeks } & Mean $\%$ area of mature bone \pm standard deviation & $64.9 \pm 10.35$ & $52.1 \pm 7.88$ & \multirow{2}{*}{$.058^{\mathrm{ns}}$} \\
\hline & Standard error mean & 4.62798 & 3.52367 & \\
\hline \multirow[b]{2}{*}{12 weeks } & Mean $\%$ area of mature bone \pm standard deviation & $74.37 \pm 6.8$ & $63.8 \pm 9.1$ & \multirow{2}{*}{$.0718^{\text {ns }}$} \\
\hline & Standard error mean & 3.03085 & 4.96653 & \\
\hline
\end{tabular}

Significance level $p<0.05$, ns=non-significant 


\section{DISCUSSION}

The autologous bone grafting is the gold standard for the treatment of bone defects. However, due to its limitations alternative treatment strategies are required. Although allograft bone grafts and xenograft bone grafts overcome the disadvantages of autogenous bone grafts, they also have drawbacks such as the strong possibility of rejection reactions and the potential spreading of diseases. ${ }^{(7)}$ With the development of tissue engineering, the field of the bone regeneration and repair is revolutionized. This alternative technique provides the benefits of bone grafting with lower complication rates, morbidity, and improved results ${ }^{(8,9)}$.

The results of this study revealed that all the defects which were managed by using bone marrow aspirate (the right tibial defects in both groups) showed better bone healing process all over the follow-up period as compared with the bone healing in the left tibial defects which were treated without loading of the bone marrow aspirate. This is explained by increased number of MSCs which was obtained through adding of the bone marrow aspirate into the defects, where the bone marrow is considered as an excellent source for MSCs ${ }^{(10-12)}$.

Also bone was formed at the peripheries of the defects before their centers in both right and left defects in the 2 groups. This revealed that MSCs residing close to the bone surface show stronger osteogenic potential in comparison to that of cells located in the central region of the bone defects. This was also found by some authors ${ }^{(13)}$.

At 6th and 12th weeks, the right defects in both groups had normal osteocyte appearance and arrangement. While in the left defects, the osteocytes had abnormal arrangement, and the most of them were devoid of nuclei. The mean value of percent area of the mature bone was also significantly higher in the right defects as compared with that of the left defects. This could be due to the presence of normal osteocytes that had normal nuclei in the right defects, so they were able to form bone better than those of left defects. This indicates that the adding of bone marrow aspirate to the defects enhance and improve the process of bone healing ${ }^{(14)}$

This study had limitations such as the lack of histological examination of the early periods of the bone repair, and the study did not count the MSCs after processing of BMA. However, it has strong points such as adult rabbits were used as experimental animals thus, their skeletons are mature so they have no great healing capacity compared to those in younger ages. So, that the results of this study depend on the use of the BMA more than the healing capacity of the animals. Additionally, it has the preferred comparative design of the studies.

\section{CONCLUSION}

In conclusion, the study findings indicate that the use of autologous BMA opens the door to a very exciting and promising field. The centrifugal concentration techniques provide better regenerative therapeutic outcomes over the whole BMA, but it is statistically insignificant.

\section{RECOMMENDATION}

It is recommended to do other studies that compare different protocols of BMA processing and investigate other details such as the number and concentration of cells and the concentration of the other biological factors that include growth factors and bone morphogenic proteins.

\section{REFERENCES}

1. Tollemara V, Colliera ZJ, Mohammeda MK, Leeb MJ, Ameerd GA, Reid RR. Stem cells, growth factors and scaffolds in craniofacial regenerative medicine. Genes and Diseases 2016; 3:56-71.

2. Shakoori P,Zhang Q,Le AD.Applications of Mesenchymal Stem Cells in Oral and Craniofacial Regeneration. J Oral Maxillofac Surg 2017; 29: 19-25.

3. Fontan FR, Piuzzi NS, Chahla J, Payne K, Robert F, LaPrade, Muschler GF, Garrido CP. Stem and Progenitor Cells for Cartilage Repair: Source, Safety, Evidence and Efficacy. Operative Techniques in Sports Medicine. OTSM 2017; 25: 25-33 
4. Kazunori S, Yu M, Wataru A, Ryosuke N, Hiromichi F, David H A, Alberto G, Keisuke K, Shuji H, Konsei S, Hideki Y, Norimasa N. Osteochondral Repair Using a ScaffoldFree Tissue-Engineered Construct Derived from Synovial Mesenchymal Stem Cells and a Hydroxyapatite-Based Artificial Bone. Tissue Eng Part A 2014; 20: 304- 2291.

5. Thua TL, Pham DN, Lê KL, Minh-Tuan Lê, Nguyen QTQ, Nguyen PH, Tran NV, Nguyen NG, Boeckx W. Tissue engineering using mesenchymal stem cell with periosteal wrap for bone defect repair in rabbits. Plast Aesthet Res $2015 ; 2: 340-345$.

6. Shapiro SA, Kazmerchak SE, Heckman MG, Zubair AC, O'Conno MI. A Prospective, Single-Blind, PlaceboControlled Trial of Bone Marrow Aspirate Concentrate for Knee Osteoarthritis. Am J Sports Med 2017; 45: 82-90.

7. Baheiraei N, Nourani MR, Mortazavi SMJ, Movahedin M, Eyni H, Bagheri F, Norahan MH, Development of a bioactive porous collagen/ $\beta$-tricalcium phosphate bone graft assisting rapid vascularization for bone tissue engineering applications . J Biomed Mater Res 2018; 106: 73-85.

8. Jeznach O, Gajc M, Korzeb K, Kłos A, Orliński K, Stępień R, Borkowicz MK, Rumian L, Pietryga K, Reczyńska K, Pamuła E, Pawlak DA New calcium-free Na2O-A12O3$\mathrm{P} 2 \mathrm{O} 5$ bioactive glasses with potential applications in bone tissue engineering. J Am Ceram Soc 2018; 101:602-611.

9. Sattary M, Khorasani MT, Rafienia M, Rozve HS. Incorporation of nanohydroxyapatite and vitamin D3 into electrospun PCL/Gelatin scaffolds: The influence on the physical and chemical properties and cell behavior for bone tissue engineering. Polym Adv Technol 2018; 29: 451-462.

10. Christoph N, Dirk H, Caroline S, Katrin S, Jo FS, Svenja H, John H B, Ingo M, Johannes F. Treatment of Large Bone Defects with a Vascularized Periosteal Flap in Combination with Biodegradable Scaffold Seeded with Bone MarrowDerived Mononuclear Cells: An Experimental Study in Rats. Tissue Eng Part A 2016; 22: 133-141.

11. Torres J, Gutierres M, Atayde L, Cortez P, Lopes MA, Santos JD, Cabral AT, VanEck CF. The benefit of bone marrow concentrate in addition to a glass-reinforced hydroxyapatite for bone regeneration: An in vivo ovine study. J Orthop Res 2017; 35: 1176-1182.

12. Shenoy RM, Pinto D, Dinesh KVN, Chandra G. Osteoinduction using autologous bone marrow in difficult orthopaedic problems - A clinical study. IJBAR 2014; 5: 288-291.

13. Siclari VA, Zhu J, Akiyama K, Liu F, Zhang X, Chandra A, Nah HD, Shi S, Qin L. Mesenchymal progenitors residing close to the bone surface are functionally distinct from those in the central bone marrow. Bone 2014; 53: 575-586

14. Hauser RA, Eteshola E. Rationale for Using Direct Bone Marrow Aspirate as a Proliferant for Regenerative Injection Therapy (Prolotherapy). OSCJ Journal 2013; 3:7-14. 